$\begin{array}{cl}\begin{array}{c}\text { Revue } \\ \text { de } / \text { histoire } \\ \text { des religions }\end{array} & \text { Revue de l'histoire des religions } \\ & \begin{array}{l}1 \mid 2014 \\ \text { Varia }\end{array}\end{array}$

\title{
Poimandrès, figure d'autorité dans la tradition hermétique
}

Poimandrès, an authority figure in the Hermetic tradition.

Anna Van den Kerchove

\section{OpenEdition}

1 Journals

Édition électronique

URL : http://journals.openedition.org/rhr/8192

DOI : $10.4000 /$ rhr.8192

ISSN : 2105-2573

Éditeur

Armand Colin

Édition imprimée

Date de publication : 1 mars 2014

Pagination : 27-46

ISBN : 978-2200929107

ISSN : 0035-1423

Référence électronique

Anna Van den Kerchove, "Poimandrès, figure d'autorité dans la tradition hermétique », Revue de

I'histoire des religions [En ligne], 1 | 2014, mis en ligne le 01 mars 2017, consulté le 19 avril 2019. URL

http://journals.openedition.org/rhr/8192 ; DOI : 10.4000/rhr.8192 


\section{Poimandrès, figure d'autorité dans la tradition hermétique}

Le premier écrit du Corpus hermeticum est intitulé Poimandrès, d'après l'être divin éponyme qui tient le rôle principal dans cet écrit; il relate la révélation cosmologique et anthropologique que Poimandrès délivre à un narrateur anonyme. Le nom Poimandrès, rare, paraît spécifique à la littérature hermétique. Après avoir fait le point sur les différentes hypothèses étymologiques scientifiques avancées pour expliquer le nom, l'intérêt se porte sur la manière dont ce nom légitime l'écrit, sur sa réception, en particulier dans la tradition hermétique, et enfin sur son rôle dans l'économie de la révélation, à partir de l'étude de trois extraits hermétiques (CH I 1-4, I 30 et XIII 15).

\section{Poimandrès, an authority figure in the Hermetic tradition.}

The first text of the Corpus Hermeticum is entitled Poimandres, according to the eponymous divine being who plays the main role in it; it narrates the cosmological and anthropological revelation that Poimandres delivers to an anonymous narrator. The name Poimandres is rare and seems specific to Hermetic literature. After an update on the various scholarly etymological hypotheses, the focus is placed on the way the name legitimises the writing, its reception in the Hermetic tradition and finally on its role in the economy of the revelation with a study of three Hermetic passages (CH I 1-4, I 30 and XIII 15). 
Le Corpus hermeticum (ci-après $\mathrm{CH}$ ), constitué probablement avant le philosophe byzantin du $\mathrm{XI}^{\mathrm{e}}$ siècle, Michel Psellus, rassemble 17 textes grecs, écrits au cours du $\mathrm{II}^{\mathrm{e}}$ et du $\mathrm{III}^{\mathrm{e}}$ siècle, qui mettent en scène Hermès Trismégiste et/ou ses disciples et qui proposent une voie de sagesse mêlant traditions grecque et égyptienne. Le premier écrit du Corpus, intitulé dans la tradition manuscrite Poimandrès d'après l'être divin éponyme dont il est question, serait l'un des plus anciens écrits hermétiques conservés, avec une datation qui varie entre la fin du $\mathrm{I}^{\mathrm{er}}$ siècle ${ }^{1}$ et le milieu du $\mathrm{II}^{\mathrm{e}}$ siècle $^{2}$. Bien que ce texte fasse partie de la littérature et de la tradition hermétique, il s'en distingue par un certain nombre de traits: il n'est question d'aucun des personnages habituellement présents (Tat, Asclépios, Ammon, Isis, Horus) ni d'Hermès Trismégiste (même si au moins une partie de la tradition hermétique représentée par l'auteur de $\mathrm{CH}$ XIII 15, probablement au $\mathrm{III}^{\mathrm{e}}$ siècle, l'a identifié au narrateur anonyme), et à leur place, l'auteur du Poimandrès met en scène un «je» anonyme et Poimandrès; la forme dialoguée, commune à la plupart des écrits hermétiques, est ici enchâssée dans une narration, avec un aspect autobiographique absent ailleurs et une fonction différente de celle des autres écrits. En effet, $\mathrm{CH}$ I relate à la fois l'instruction apocalyptique - au contenu à la fois cosmogonique, anthropogonique et anthropologique - délivrée par Poimandrès au narrateur anonyme et les conséquences de la révélation pour ce dernier (sa transformation interne et son investiture en tant que guide salvateur). $C H$ I apparaît ainsi comme un «texte fondateur», au sens où l'auteur met en place une nouvelle voie de salut, fondée sur l'acquisition de connaissances et sur l'adoption d'un mode

1. Charles H. Dodd, The Bible and the Greeks, London, Hodder and Stoughton, $1954^{2}$ (1935), p. 99 n. 1, p. 203 et p. 209; Garth Fowden, Hermès l'Égyptien. Une approche historique de l'esprit du paganisme tardif, traduction: J.-M. Mandosio, Paris, Les Belles Lettres, 2000 (1986 pour l'édition anglaise) («L'âne d'or»), p. 29 n. 53.

2. Cette datation a été nouvellement proposée suite à une remarque d'Alberto Camplani lors du colloque «Dévots, fidèles de d/Dieu», le 28 novembre 2008; $c f$. Anna Van den Kerchove, «Les hermétistes et les conceptions traditionnelles des sacrifices », dans Nicole Belayche et Jean-Daniel Dubois (éd.), L'Oiseau et le poisson. Cohabitations religieuses dans les mondes grec et romain, Paris, Presses de l'Université Paris Sorbonne, 2011 («Religions dans l'histoire»), p. 61-81, part. p. 80 . 
de vie, et où cet écrit a été perçu comme étant à l'origine d'une tradition philosophico-religieuse ${ }^{3}$.

Le nom Poimandrès identifie un être que la description désigne clairement comme un être divin: taille immense, «mesure illimitée », polymorphie ( $\mathrm{CH}$ I 4), identification au premier principe qui est l'Intellect, lui-même identique à la Lumière ( $\mathrm{CH}$ I 6), et omniscience ( $\mathrm{CH}$ I 2). Quelles sont les fonctions de ce nom divin dans l'économie du texte, et plus particulièrement de la révélation? Répondre à cette question nous amène à s'interroger sur l'autorité du texte, sur l'étymologie du nom et sur son usage dans l'écrit, en se plaçant aussi bien du point de vue de l'auteur que de celui des bénéficiaires.

\section{LE NOM DIVIN：SIGNATURE DE LA RÉVÉLATION}

Pour de nombreux écrits antiques, il est possible de parler du «silence sur les auteurs» ou du «silence des auteurs $»^{4}$ sur euxmêmes. C'est le cas des écrits hermétiques et notamment de $C H \mathrm{I}$, même si du fait de la narration à la première personne, narrateur et auteur semblent se confondre.

Face à l'auteur qui se pose plus comme un scribe et face à un narrateur anonyme, le fait que l'être divin soit le seul à être nommé le désigne comme le fondement de la légitimité et de l'autorité des énoncés rapportés et de l'écrit. La mention de son nom permet d'identifier la source divine et, en quelque sorte, de signer l'écrit d'une signature divine.

3. Certes, il est difficile d'affirmer que tous les auteurs des écrits hermétiques connaissaient $\mathrm{CH}$ I; néanmoins, il existe des témoignages attestant que Poimandrès a été perçu comme étant à l'origine d'une révélation apocalyptique et salvatrice; c'est le cas en particulier de $\mathrm{CH}$ XIII 15 et peut-être du Compte final I 8 de Zosime, dont il sera question plus bas. De plus, il n'est peut-être pas anodin que le traité ait été placé en tête du Corpus hermeticum par le compilateur anonyme.

4. Gérard Leclercq, Histoire de l'autorité. L'assignation des énoncés culturels et la généalogie de la croyance, Paris, Presses universitaires de France, 1996 («Sociologie d'aujourd'hui»), p. 32. 


\section{UN NOM RARE ET HERMÉTIQUE}

La question se pose alors du choix du nom, car il est rare. Dans la tradition hermétique, en dehors des occurrences de $\mathrm{CH} \mathrm{I}$, il n'apparait que trois fois: l'auteur de $\mathrm{CH}$ XIII (probablement du III $^{\mathrm{e}}$ siècle), mentionne deux fois Poimandrès ( $\mathrm{CH}$ XIII 15) et l'identifie à l'instructeur d'Hermès Trismégiste; vers 300, Zosime de Panopolis, dans le Compte final 8, le mentionne sous la forme Poimenandra (à l'accusatif) en relation avec le baptême de l'intellect (il s'agit d'une allusion à $C H \mathrm{IV}^{5}$ ).

En dehors de la tradition hermétique, le nom est cité uniquement dans des citations d'extraits hermétiques: une occurrence chez Fulgence vers 500 (Mitologiarum, libri VIII, XV. Fabula de novem Musis 48), une autre dans une collection syriaque d'oracles grecs de la fin du VI ${ }^{\mathrm{e}}$ ou du début du VII ${ }^{\mathrm{e}}$ siècle (où l'oracle $S y r-A 21$ cite, en les christianisant, quelques extraits de $C H$ XIII 1-46) et plusieurs occurrences dans l'œuvre de Michel Psellus'. Dans le reste de la littérature grecque conservée à l'heure actuelle, le nom propre grec le plus proche est celui de Poimandros ${ }^{8}$, qui pourrait être un composé de poimèn et de anèr et qui est porté uniquement par deux personnages, l'un historique ${ }^{9}$, l'autre mythique ${ }^{10}$. Rapprocher

5. Voir les textes infra.

6. Voir Sebastian Brock, «A Syriac Collection of Prophecies of the Pagan Philosophers», Orientalia Lovaniensia Periodica, 14, 1983, p. 203-246 et part. p. 203-210 pour la présentation générale, et Idem, «Some Syriac Excerpts from Greek Collections of Pagan Prophecies », Vigiliae Christianae, 38, 1984, p. 77-90.

7. Michel Psellus, Orationes forenses 1.894; Oratioria minora 24.93; Opuscula psychologica 154-155.

8. La ressemblance avec ce nom a été relevée par Frank Granger, «The Poemandres of Hermes Trismegistus », The Journal of Theological Studies, 5, 1904, p. 400 et Walter Scott, Hermetica: the Ancient Greek and Latin Writings Which Contain Religious or Philosophic Teachings Ascribed to Hermes Trismegistus, vol. 2, London, Dawsons, 1924, p. 15.

9. Il s'agit de Poimandros le Mélien; cf. Anthologie palatine VII, épigramme funéraire 171.

10. Plusieurs sources mentionnent un héros contemporain de l'expédition grecque contre Troie, Poimandros, fondateur de Poimandria (ancien nom de Tanagra) et meurtrier de l'un de ses fils: Strabon, Géographie IX, 2.10 (avec

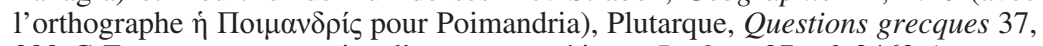
299 C-E et un commentaire d'un texte poétique, P. Oxy. 27, n 2463 (papyrus du II $^{\mathrm{e}}$ ou $\mathrm{III}^{\mathrm{e}}$ siècle) dans The Oxyrhynchus Papyri: Part XXVII, éd., traduction et notes de Eric G. Turner, John R. Rea, L. Koenen et José, M. Fernández Pomar, Londres, Egypt Exploration Society, 1962. Voir aussi Wilhelm. Pape et Gustav Benseler, Wörterbuch der Griechischen Eigennamen. Nachdruck der dritten Auflage. Zweiter Band [L-Omega], Graz, Akademische Druck- u. Verlagsanstalt, 1959, p. 1216 b: le nom est traduit par «chasseur» (Weidmann). 
Poimandrès de Poimandros est cependant difficile: la finale -ès reste inexpliquée phonétiquement, et les sources antiques n'attestent aucun rapport entre les deux Poimandros et Poimandrès. Ce dernier nom a ainsi pu être inventé par l'auteur de $C H$ I ou être repris d'une source antérieure méconnue, ce qui laisse toujours ouverte la question des raisons qui ont motivé l'auteur. Il avait à sa disposition beaucoup de noms divins, parfois «étranges", qui pouvaient tout aussi bien assurer l'autorité des énoncés, en évoquant notamment explicitement des sagesses orientales. Une explication peut être apportée par la signification étymologique du nom dont l'auteur aurait eu connaissance (en particulier si le nom est son invention). Il faut pour cela se tourner du côté des étymologies scientifiques.

\section{ÉTYMOLOGIES MODERNES}

Dès le $\mathrm{XVI}^{\mathrm{e}}$ siècle, les savants ont cherché à élucider la signification étymologique de ce nom, en ayant recours au grec, au copte ou à l'égyptien ancien ${ }^{11}$. Nous donnons par ordre chronologique les différentes propositions dans le tableau suivant: $:^{12131415}$

\begin{tabular}{|c|c|c|c|}
\hline Étymologie & Signification & $\begin{array}{l}\text { Savant qui } \\
\text { proposa } \\
\text { l'hypothèse }\end{array}$ & $\begin{array}{c}\text { Savants qui acceptent } \\
\text { l'hypothèse }\end{array}$ \\
\hline $\begin{array}{l}\text { poimèn }+ \\
\text { anèr }\end{array}$ & $\begin{array}{l}\text { le berger des } \\
\text { hommes }\end{array}$ & $\begin{array}{l}\text { Isaac } \\
\text { Casaubon }^{12}\end{array}$ & $\begin{array}{l}\text { Richard Reitzenstein }{ }^{13} \text {, } \\
\text { Ernst Haenchen }^{14} \text {, } \\
\text { Birgen A. Pearson }^{15}\end{array}$ \\
\hline
\end{tabular}

11. Seul Peter Kingsley émit l'idée d'une double étymologie: «Poimandres: The Etymology of the Name and the Origins of the Hermetica», dans Roelof van den Broek et Cis van Heertum (éd.), From Poimandres to Jacob Böhme: Gnosis, Hermetism and the Christian Tradition, Amsterdam, In de Pelikaan, 2000, p. 41-76, part. p. 45 ; cet article est la réimpression, avec quelques modifications, de la première version parue dans Journal of Warburg and Courtauld Institutes, 56, 1993, p. 1-24.

12. Isaac Casaubon, Cohortationes in Gentiles 57, 1614, cité par Richard Marcus, «The Name Poimandres», Journal of Near Eastern Studies, 8.1, 1949, p. $40-43$, part. p. 40.

13. Richard Reitzenstein, Poimandres. Studien zur griechisch-ägyptischen und frühchristlichen Literatur, Leipzig, Teubner, 1904, p. 11-12 et p. 32.

14. Ernst Haenchen, «Aufbau und Theologie des Poimandres», dans idem, Gott und Mensch. Gesammelte Aufsätze I, Tübingen, Mohr, 1965, p. 335-377, part. p. 335.

15. Birgen A. Pearson, «Jewish Elements in Corpus Hermeticum I (Poimandres)», dans Roelof Van Den Broek et Maarten J. Vermaseren (éd.), Studies in Gnosticism and Hellenistic Religions: presented to Gilles Quispel on the Occasion of his 65th Birthday, Leiden, Brill, 1981, p. 336-348, part. p. 340 n. 12. 


\begin{tabular}{|c|c|c|c|}
\hline Étymologie & Signification & $\begin{array}{c}\text { Savant qui } \\
\text { proposa } \\
\text { l'hypothèse }\end{array}$ & $\begin{array}{c}\text { Savants qui acceptent } \\
\text { l'hypothèse }\end{array}$ \\
\hline p-mntre & le témoin & Frank Granger ${ }^{16}$ & Peter Hofrichter ${ }^{17}$ \\
\hline p-eimen-rè & $\begin{array}{l}\text { la } \\
\text { connaissance } \\
\text { du dieu soleil }\end{array}$ & $\begin{array}{l}\text { Francis } \\
\text { Llewellyn } \\
\text { Griffith }^{18}\end{array}$ & $\begin{array}{l}\text { Walter Scott }{ }^{19} \text {, Charles } \\
\text { H. Dodd }\end{array}$ \\
\hline $\begin{array}{l}p \text {-eimen-t- } \\
\text { mntero }\end{array}$ & $\begin{array}{l}\text { la raison de la } \\
\text { souveraineté }\end{array}$ & Ralph Marcus ${ }^{21}$ & \\
\hline Maâ.t-rè & Vérité de Ré & $\begin{array}{l}\text { Heinz } \\
\text { J. Thissen }\end{array}$ & \\
\hline p-eimentre-rè & $\begin{array}{l}\text { la } \\
\text { connaissance } \\
\text { du dieu soleil }\end{array}$ & Peter Kingsley ${ }^{23}$ & \\
\hline  & $\begin{array}{l}\text { Pharaon de la } \\
\text { Maât de Ré }\end{array}$ & $\begin{array}{l}\text { Howard } \\
\text { M. Jackson }{ }^{24}\end{array}$ & \\
\hline
\end{tabular}

La première hypothèse, la seule à être fondée sur le grec, a été avancée par celui-là même qui mit en cause l'ancienneté des écrits hermétiques, le Genevois protestant Isaac Casaubon. Ce dernier considère que le nom dérive de poimèn et il le compare à Jean 10.14 - ce rapprochement est lié à son idée que les textes hermétiques ont été écrits par des chrétiens. Cette hypothèse rejoint les interprétations antiques qui, comme nous le verrons plus bas, se fondent surtout sur des similitudes homophoniques. Cependant, elle laissait inexpliquées la présence du delta et la finale.

16. Frank Granger, «The Poemandres», art. cit., p. 400.

17. Peter Hofrichter, "Gnosis und ägyptische Mythologie», dans Manfred Görg et Günther Hölbl (éd.), Ägypten und der östliche Mittelmeerraum im 1. Jahrtausend v. Chr. Akten des interdisziplinären Symposions am Institut für Ägyptologie der Universität München 25-27.10.1996, München, Harrassowitz, 2000, p. 101-118, part. p. 116.

18. Elle a été transmise par Walter Scott, Hermetica, op. cit., p. 16.

19. Ibidem.

20. Charles H. Dodd, The Bible, op. cit., p. 99 n. 1.

21. Ralph Marcus, «The Name», art. cit., p. 40-43.

22. Heinz J. Thissen, «Demotistik und Ägyptologie. Anmerkungen zu demotischen literarischen Texten», Zeitschrift für ägyptische Sprache und Altertumskunde, 117, 1990, p. 63-69, part. p. 66.

23. Peter Kingsley, «Poimandres», art. cit., p. 41-76.

24. Howard M. Jackson, «A New Proposal for the Origin of the Hermetic God Poimandres», Zeitschrift für Papyrologie und Epigraphik, 128, 1999, p. 95-106. 
$\mathrm{Au} \mathrm{Xx}^{\mathrm{e}}$ siècle, l'intérêt renaît pour le nom, avec l'étymologie grecque qui revient régulièrement sur le devant de la scène, et de nouvelles hypothèses à partir de l'égyptien, le copte ou l'égyptien ancien. Deux questions se posent: l'une concerne la phonétique (vocalisation, morphème final -rès et présence du phonème $d$ ), l'autre le lien entre le nom et le contexte apocalyptique de $\mathrm{CH}$ I. La seconde question fut résolue plus facilement, et chaque savant a montré comment son hypothèse s'insérait bien dans le contexte de l'écrit. En revanche, l'argumentation fut plus difficile à mener pour ce qui concerne la phonétique. Ainsi, Frank Granger et Ralph Marcus n'expliquent pas la finale, et leur étymologie a été justement rejetée ${ }^{25}$. Walter Scott ne transmet pas l'argumentation de Francis Llewellyn Griffith, et Heinz J. Thissen ne détaille pas son affirmation que Poimandrès n'est autre que la Maât de Rê ce n'est pas non plus le propos de son article. Peter Kingsley et Howard M. Jackson proposent une argumentation plus détaillée. Le premier affirme à juste titre que le morphème rès est la transcription grecque standard pour les noms égyptiens se terminant avec le nom de $\mathrm{Re}^{26}$ et il modifie l'étymologie proposée par Francis Llewellyn Griffith, p-eime n-rè en p-eime nte-rè, afin de mieux rendre compte de la présence du phonème $d$. Le second suit Heinz $\mathrm{J}$. Thissen ${ }^{27}$ et, contrairement à ses autres prédécesseurs, estime que Poimandrès n'est pas une création artificielle mais dérive de $\operatorname{Pr}$-âa-ni-maâ.t-rè («Pharaon qui appartient à la Maât de Ré»). Ce nom est celui sous lequel le pharaon de la XII ${ }^{\mathrm{e}}$ dynastie, Amenemhat III, a été divinisé et vénéré; son culte est toujours vivant aux époques hellénistique et gréco-romaine, en particulier au Fayoum ${ }^{28}$, et le nom a été

25. Walter Scott, Hermetica, op. cit., p. 16 n. 9; Peter Kingsley, «Poimandres», art. cit., p. 47 et n. 15 .

26. Peter Kingsley, «Poimandres», art. cit., p. 47. Pour le problème de la transcription grecque du 'ayin égyptien, voir John D. Ray, «Nasal Vowels in Egyptian», Enchoria, 8.2, 1978, p. 77-78, part. p. 77; pour quelques exemples, voir Willy Peremans et Edmond Van't Dack, Prosopographia Ptolemaica. VII. Index Nominum, Louvain, Peeters, 1975 («Studia Hellenistica» 20), p. 26.

27. Howard M. Jackson, «A New Proposal», art. cit., p. 100-101, 105.

28. Sur le culte de ce pharaon, voir: Otto Rubensohn, «Pramarres», Zeitschrift für ägyptische Sprache und Altertumskunde, 42, 1905, p. 111-115 + Tafel VI; Henry Riad, «Le culte d'Amenemhat III au Fayoum à l'époque ptolémaïque», Annales du Service des Antiquités de l'Égypte, 55, 1958, p. 203-206, part. p. 204-5; Edda Bresciani, «Iconografia e culto di Premarres nel Fayum», Egitto e Vicino Oriente, 9, 1986, p. 49-58, part. p. 49-50; Winfried J. R. Rübsam, Götter und Kulte in Faijum während der griechisch-römisch-byzantinischen Zeit, Bonn, Habelt, 
différemment transposé en grec. Howard M. Jackson dresse une liste de ces attestations et considère que la forme donnée par le quatrième hymne d'Isidore à Isis, Porramanrès, est la plus proche de Poimandrès; mais il n'explique pas le lien entre porra- et poi-.

Parmi toutes les hypothèses avancées jusque-là, la suggestion de Peter Kingsley, qui modifie l'hypothèse de Francis Llewellyn Griffith, est la plus probante; de même, le rapprochement avec maâ.t-rè opéré par Heinz J. Thissen puis par Howard M. Jackson est à prendre en compte, sans suivre jusqu'au bout ce dernier - ses arguments pour la première partie du nom ne convainquent pas totalement, et sa conclusion sur l'auteur de $C H$ I comme dévot du culte d'Amenemhat III et sur la provenance fayoumique de l'écrit hermétique mériterait d'être approfondie ${ }^{29}$. Cela ne signifie pas que de nouvelles recherches ne pourraient pas permettre de formuler d'autres hypothèses.

\section{FAIRE DE L'ÉCRIT HERMÉTIQUe UN LIVRE DE THOT}

Que l'on accepte l'étymologie suggérée par Heinz J. Thissen et suivie en partie par Howard M. Jackson, ou celle de Peter Kingsley qui améliore la suggestion de Francis Llewellyn Griffith, Poimandrès serait lié au dieu égyptien Rê: dans le premier cas, il est lié à sa Maât, que l'on peut traduire de manière approximative par «vérité», «ordre» et «norme»; dans le second cas, il en est la connaissance. Peter Kingsley renforce le lien avec Rê en considérant que l'expression ho tès authentias nous («l'intellect de la Souveraineté») - qui qualifie Poimandrès en $\mathrm{CH}$ I 2 et en $\mathrm{CH}$ XIII 15 - est l'interprétation grecque de Poimandrès-«La connaissance

1974. Voir aussi Ghislaine Widmer, «Pharaoh Maâ-Rê, Pharaoh Amenemhat and Sesostris: three Figures from Egypt's Past as seen in Sources of the Graeco-Roman Period», dans Kim Ryholt (éd.), Acts of the Seventh International Conference of Demotic Studies. Copenhagen, 23-27 August 1999, Copenhagen, Museum Tusculanum Press, 2002, p. 377-393.

29. L'auteur n'explique pas le passage $P r$-âa de à poi. Faut-il rapprocher cette syllabe de l'article copte dit fort, intoné pai ou désintoné pei, dérivant du démonstratif masculin égyptien pzy-? Mais comment expliquer le passage de peià poi-? Sa conclusion selon laquelle l'auteur hermétique est un dévot du culte d'Amenemhat III et que le traité hermétique provient probablement du Fayoum nous paraît hâtive et n'est, pour le moment, étayée par aucun autre élément, ni textuel ni archéologique. 
de Rê» et que la Souveraineté correspondrait au dieu égyptien $\mathrm{R} \hat{\mathrm{e}}^{30}$; la Souveraineté serait en quelque sorte une interpretatio graeca ou une interpretatio hermétique de Rê. Il est vrai qu'il existe des similitudes entre le dieu égyptien et la Souveraineté hermétique: le rapport à la lumière, l'équivalence entre d'un côté les «organes» de Ré ou les capacités de la Souveraineté absolue et de l'autre des divinités. Seul le nombre de ces divinités change: pour la Souveraineté absolue, seulement Poimandrès, pour Ré, Horus et Thot. Dans la tradition égyptienne, Horus est le cœur de Ré, cœur qui est la compréhension ou la perception personnifiée par $\mathrm{Sia}^{31}$ et qui est l'équivalent de l'intellect hermétique ${ }^{32}$; Thot est la langue ou la parole de Ré, qui sont l'expression ou la parole créatrice personnifiée par $\mathrm{Hou}^{33}$. Thot pourrait ainsi être l'équivalent égyptien de l'expression ho tès authentias logos («la parole de la Souveraineté») apposée à Poimandrès en $\mathrm{CH}$ I 30. Cependant, comme Peter Kingsley l'a remarqué, Thot pourrait aussi être désigné par l'expression ho tès authentias nous, car il est souvent associé à $\mathrm{Sia}^{34}$. De plus, le syntagme «cœur de Ré» est une épithète de Thot fréquente à l'époque gréco-romaine ${ }^{35}$, et, dans

30. Peter Kingsley, «Poimandres», art. cit., p. 48-50. L'auteur reprend l'idée de Francis Llewellyn Griffith rapportée par Walter Scott, tout en l'étayant par des arguments supplémentaires.

31. Sur Sia et Hou, voir Alan H. Gardiner, «Some Personifications. II. Hu, "Authoritative Utterance". Sia, "Understanding"», Proceedings of the Society of Biblical Archaeology, 38, 1916, p. 43-54 et p. 83-95. Voir également les Textes des Sarcophages, en particulier les formules 321, 335, 647 et 689 .

32. En effet, pour les auteurs anciens, le cœur est le siège de l'intellect ou l'intellect lui-même. Cf. Georges Posener, «Cœur», dans Serge Sauneron et Jean Yoyotte, Dictionnaire de la civilisation égyptienne, Paris, F. Hazan, 1959, p. 60-61. Michel Tardieu, Codex de Berlin: écrits gnostiques, Paris, Cerf, 1984 («Sources gnostiques et manichéennes»1), p. 232. Origène, Fragmenta in evangelium Joannis 13.16.

33. Rappelons également que Thot est le dieu de la parole et des écrits; voir l'hymne à Thot de la statue $\mathrm{n}^{\circ} 2293$ du musée de Berlin dans Boris von Turajeff, «Zwei Hymnen an Thoth», Zeitschrift für ägyptische Sprache und Altertumskunde, 33, 1895, p. 120-125, part. p. 123. Patrick Boylan, Thot, the Hermes of Egypt. A Study of some Aspects of Theological Thought in Ancient Egypt, Oxford, 1922, p. 92-101. Christophe Leitz et alii, Lexikon der ägyptischen Götter und Götterbezeichnungen, Band VII, Louvain, Peeters, 2002 ( Orientalia Lovaniensia Analecta»116), p. 640 a et b, «Ikonographie» A d et E 2 c.

34. Peter Kingsley, «Poimandres», art. cit., p. 51; Patrick Boylan, Thot, op. cit., p. 103-106.

35. Marie-Thérèse Derchain-Urtel, Thot à travers ses épithètes dans les scènes d'offrandes des temples d'époque gréco-romaine, Bruxelles, 1981 («Rites 
des textes égyptiens, Sia-compréhension et Hou-expression sont réunis en Thot $^{36}$, de la même manière que la Parole et l'Intellect de la Souveraineté le sont en Poimandrès. Le rapprochement entre Poimandrès et Thot n'est pas non plus absent si l'on accepte le lien entre Poimandrès et la Maât de Rê, car Thot est le dieu égyptien le plus régulièrement mis en rapport avec la Maât ${ }^{37}$. Ainsi, Poimandrès pourrait être l'interpretatio graeca ou hermétique de Thot, luimême compréhension, parole et remplaçant de Rê en son absence.

Or, les Égyptiens attribuaient à Thot les livres sacrés et remplis de sagesse conservés dans la «Maison de vie» des temples, les «livres de Thot». Écrits en caractères hiéroglyphiques ou $m d w n t \underline{t r}$ («les paroles du dieu»), ils remplacent la parole de Rê devenue inaudible et sont parfois appelés «b3w de Rê», $b_{3} w$ étant souvent traduit par «émanations $»^{38}$. Ce lien permettrait de rattacher la révélation de Poimandrès à ces écrits et d'un faire un nouveau «livre de Thot», avec tout ce que cela implique en termes d'autorité et de légitimité: l'ensemble du contenu du traité bénéficie de l'aura des «livres de Thot »; cela concerne à la fois la révélation délivrée par Poimandrès,

Égyptiens» III), p. 81-82. Voir Christophe Leitz et alii, Lexikon der ägyptischen Götter und Götterbezeichnungen, Band vi, Louvain, Peeters, 2002 («Orientalia Lovaniensia Analecta» 115), p. 642 b, «Funktionen» P a et b; Esna III, 206.11 (Serge Sauneron, Le Temple d'Esna [n॰ 194-398], Le Caire, IFAO, 1968 [«Esna» III] et un commentaire du même auteur : «Remarques de philologie et d'étymologie [en marge des textes d'Esna]», dans Jean Sainte Fare Garnot, Mélanges Mariette, Le Caire, IFAO, 1961 [«Bibliothèque d'Étude» 32], p. 229-249, part. p. 234-235) et Esna V 219 (Serge Sauneron, Les Fêtes religieuses d'Esna aux derniers siècles du paganisme, Le Caire, IFAO, 1962 [«Esna» V]).

36. François Daumas, «Le sanatorium de Dendara», Bulletin de l'Institut Français d'Archéologie Orientale, 56 1957, p. 35-57, part. p. 45. Voir aussi MarieThérèse Derchain-Urtel, Thot à travers ses épithètes, op. cit., p. 81-94.

37. Patrick Boylan, Thot, op. cit., p. 88-91; Bernadette Menu, «Le tombeau de Pétosiris (2). Maât, Thot et le droit», Bulletin de l'Institut Français d'Archéologie Orientale, 95, 1995, p. 280-295. Voir aussi Marie-Thérèse Derchain-Urtel, Thot à travers ses épithètes, op. cit.

38. Voir le Mythe de la Vache du Ciel, 237-250 et Erik Hornung, Der ägyptische Mythos von der Himmelskuh. Eine Ätiologie des Unvollkommenen, Göttingen, Vandenhoeck \& Ruprecht, 1982, p. 45. Voir aussi la Stèle de la famine 5 et Myriam Lichtheim, Ancient Egyptian Literature, I, Berkeley, University of California Press, 1973, p. 101 n. 8; Michèle Broze, «La réinterprétation du modèle hiéroglyphique chez les philosophes de langue grecque», dans Lucia Morra et Carla Bazzanella (éd.), Philosophers and Hieroglyphs, Torino, Rosenberg \& Sellier, 2003, p. 37-51, part. p. 40; Philippe Derchain, Le Papyrus Salt 825 (B.M. 10051), rituel pour la conservation de la vie en Égypte, Bruxelles, Palais des Académies, 1965, p. 19. 
l'investiture, par ce dernier, du narrateur en guide salvateur et les discours que le narrateur devenu guide délivre ultérieurement.

\section{LA RÉCEPTION DU NOM PoIMANDRÈS}

Qu'une autorité ait été reconnue à Poimandrès semble avoir été acceptée au moins par une partie de la tradition hermétique; l'auteur de $C H$ XIII 15 en témoigne, ainsi que Zosime, qui intégra des éléments hermétiques dans ses écrits. Certes, les autres écrits hermétiques sont silencieux sur le nom; soit ils l'ignorent, soit ils ne voient peut-être pas l'utilité de le mentionner. En effet, en $\mathrm{CH}$ I comme en $\mathrm{CH}$ XIII, le nom est mentionné à des moments spécifiques de la révélation, comme nous le verrons un peu plus loin.

Les lecteurs de $\mathrm{CH}$ I - d'autres auteurs hermétistes et les bénéficiaires de l'écrit - avaient-ils cependant conscience que le nom Poimandrès permettait de rattacher la révélation à un écrit de Thot? Percevaient-ils le lien entre le nom et l'expression «l'Intellect de la Souveraineté » si l'on accepte l'hypothèse de Peter Kingsley? Ernst Haenchen objecta que cela était impossible, d'où le recours à l'hypothèse étymologique grecque ${ }^{39}$. Toutefois, que le lecteur ne puisse pas saisir une étymologie ne prouve pas l'absence de ladite étymologie. De plus, à la suite de Peter Kingsley, nous pensons qu'une double étymologie peut être envisagée, l'une égyptienne et originelle, l'autre, grecque et secondaire. Mêlant traditions égyptienne et grecque, comme la plupart des auteurs hermétistes, l'auteur de $C H \mathrm{I}$ a pu vouloir s'adresser aussi bien à un Grec qu'à un Égyptien hellénisé et avoir choisi en partie ce nom parce qu'il y avait une homophonie avec poimèn, le berger.

La réception de ce nom dans des textes antiques montre en effet que cette homophonie a été perçue. Le plus ancien témoignage antique attestant de la réception de ce nom en dehors de $\mathrm{CH}$ I est un autre texte hermétique. En $\mathrm{CH}$ XIII 15, le disciple d'Hermès, Tat, alors qu'il vient d'être régénéré, demande à son maître l'hymne des Puissances. Hermès approuve cette demande et il commence

39. Ernst Haenchen, «Aufbau», art. cit., p. 335 ; Birgen A. Pearson, «Jewish», art. cit., p. 340, n. 12. 
par préciser la manière de dire et d'écouter l'hymne. Il introduit ces indications par ces mots : «Poimandrès, l'Intellect de la Souveraineté, ne m'a pas transmis plus que ce qui est écrit, sachant que par moimême, je serai capable de tout concevoir (noein), d'entendre ce que je veux et de voir toutes les choses. » Plus loin, en $C H$ XIII 19, au cours de l'hymne, Hermès dit: «en effet, l'Intellect veille (poimainei) sur ta Parole», c'est-à-dire celle de Dieu. Le rapprochement effectué par Peter Kingsley ${ }^{40}$ entre cette proposition et le nom de Poimandrès est tentant. C'est en effet la seule occurrence du verbe poimainein, «garder, veiller sur», dans l'ensemble des écrits hermétiques, et elle semble faire écho à la mention du nom Poimandrès avant l'hymne. Il est vraisemblable que l'auteur de $C H$ XIII ait interprété la première partie du nom de Poimandrès, poim-, comme signifiant «celui qui veille sur», «le berger», tout en laissant de côté la seconde partie du nom, -andrès, laquelle ne devait probablement pas convenir au contexte de l'hymne. La proposition citée s'applique à la Parole et à l'Intellect divins, à la parole et à l'intellect humains - dans les écrits hermétiques, il y a en effet souvent une confusion volontaire entre les deux ordres humain et divin pour ce qui concerne la parole et l'intellect. L'auteur de $C H$ XIII exprime ainsi l'idée que tout intellect a une fonction de berger par rapport à la parole, qu'il la guide et que la parole est sous sa dépendance.

Cette interprétation de Poimandrès-berger semble être partagée un peu plus tard par Zosime de Panopolis. Dans son Compte final I 8, il incite Théosobie à se rendre parfaite grâce à des procédés alchimiques (les teintures) et à se rendre auprès de Dieu au moyen d'autres actions: «Lorsque tu reconnaîtras que tu as été rendue parfaite, alors, [ayant obtenu] les teintures naturelles, crache sur la matière, réfugie-toi auprès de Poiménandre (katadramousa epi ton Poimenandra), et, après avoir été baptisée dans le cratère ${ }^{41}$, élance-toi vers ta fratrie $\left(\right.$ genos $\left.^{42}\right) »$. Contrairement à la tradition

40. Peter Kingsley, «Poimandres», art. cit., p. 44. Il n'a pas été le premier à le faire: voir André-Jean Festugière et Arthur D. Nock, dans Corpus Hermeticum, texte établi par Arthur D. Nock et traduit par André-Jean. Festugière, tome II, Paris, Les Belles Lettres, 1991 (cinquième tirage de l'édition de 1946), p. 217 n. 83.

41. Il s'agit d'une allusion au traité $C H$ IV où il est question d'acquérir l'intellect en s'immergeant dans un cratère.

42. Pour le texte grec, voir André-Jean Festugière, La Révélation d'Hermès Trismégiste, vol. 1: L'Astrologie et les sciences occultes, Paris, les Belles Lettres, 1989 (réimpression de la seconde édition de 1950), p. 281 et 368. 
hermétique directe, Zosime transmet le nom de Poimandrès sous une orthographe légèrement modifiée: Poimenandra, une modification qui pourrait être imputée à une erreur de la transmission, directe ou indirecte, du nom ${ }^{43}$. Cependant, il pourrait s'agir d'une tentative pour clarifier le sens de Poimandrès en en faisant un composé de poimen(c'est-à-dire le radical alternant avec poimèn ${ }^{44}$ aux cas autres que le nominatif), auquel aurait été ajouté le radical $a n d r$-. Le lien avec le rôle de berger apparaît ainsi plus évident pour le lecteur. Si la nouvelle orthographe peut être antérieure à l'œuvre de Zosime, ce dernier l'accepte et a donc conscience de la signification du nom. Il en va de même pour Théosobie, Zosime mentionnant Poimenandre sans l'introduire; celle-ci doit se réfugier, katadramein, auprès de Poimenandre, de la même façon que l'on se réfugierait auprès d'un berger, faisant de cette figure un être qui prend soin des hommes parfaits et qui veille sur eux.

Une telle interprétation ne serait pas spécifique aux milieux hermétistes ou proches des hermétistes. Dans son ouvrage Mitologiarum XV. Fabula de novem Musis 48, écrit vers 500, le mythographe latin Fulgence introduit l'unique citation hermétique de son œuvre, un extrait de $C H$ I 1, de la manière suivante: «Et Hermès dans son livre d'Opimandra dit (et Hermes in Opimandrae libro ait): "à cause d'une satiété de nourriture ou à cause d'un corps léger" $\gg^{45}$. La transposition latine du grec poimandrès attestée ici peut être éclairée par une comparaison avec la transcription opérée par Marsile Ficin au XV $\mathrm{XV}^{\mathrm{e}}$ siècle. Dans sa traduction latine de textes hermétiques intitulée Pimander. Mercurii Trismegisti liber de sapientia et potestate dei, Marsile Ficin transcrit le nominatif Poimandrès en Pimander et le génitif Poimandrou de CH I 30 en l'ablatif Pimandro $^{46}$ : la syllabe poi est donc transcrite $p i$, et la déclinaison suivrait celle du modèle ager, -gri de la deuxième déclinaison. Chez Fulgence, nous retrouvons la syllabe pi, mais celle-ci est précédée de la lettre $o$, à la différence de Marsile Ficin.

43. C'est la position de Walter Scott, Hermetica, op. cit., vol. 4, p. 142.

44. Pierre Chantraine, La Formation des noms en grec ancien, Paris, Klincksieck, 1979, p. 170-174 et surtout p. 174.

45. Fulgence cite une expression de $C H$ I 1 avec une erreur - koufou au lieu de kopou -, avant d'en donner une explication.

46. Édition de 1505, numérisée par Gallica (référence FRBNF37251523), p. 16: «hec mihi cotingerunt ex mente haurienti. id est ex Pimandro divine poterie verbo». 
Plusieurs explications sont possibles : elle peut être le résultat d'une métathèse des lettres $o$ et $p i$, donnant ainsi opi à partir de poi; ou bien elle provient de la transcription latine de poi, comme chez Marsile Ficin. Dans ce dernier cas, Fulgence, ou sa source, aurait inclus dans le nom soit l'article grec $o^{47}$, soit l'interjection $\hat{o}$. Dans tous les cas, le début du nom latin, opi-, rappellerait le terme ops, génitif opis, dont l'un des sens est «aide», «appui» et «assistance», ce qui correspond à l'une des fonctions de Poimandrès ${ }^{48}$. Une autre différence importante avec la transposition de Marsile Ficin concerne la déclinaison. Fulgence donne la terminaison du génitif féminin $-a e$, ce qui correspondrait à un nom de la première déclinaison: Opimandra, ae. La deuxième partie du nom, -mandra, renvoie alors à un mot latin féminin signifiant «troupeau» : mandra, ae. Si toutes ces explications demeurent hypothétiques, elles tendent à montrer qu'il ne s'agirait pas d'une faute d'orthographe et que l'auteur de la transcription latine - Fulgence ou, plus vraisemblablement, un auteur antérieur mieux renseigné sur le contenu de $\mathrm{CH}$ I -, loin de s'être contenté d'une simple transcription du grec, aurait cherché à renforcer le lien de Poimandrès avec le troupeau et le berger. Pas plus que les œuvres de Michel Psellus, l'oracle syriaque Syr-A 21 ne nous apprend quelque chose, car le nom est transcrit fidèlement.

Que ces auteurs, ou leurs sources, aient mis le nom Poimandrès en relation avec le berger, et ce probablement dès le $\mathrm{III}^{\mathrm{e}}$ siècle avec l'auteur de $\mathrm{CH}$ XIII, est compréhensible, vu la manière dont Poimandrès est présenté dans $C H \mathrm{I}$, en particulier avec la troisième proposition de la réponse à la question du narrateur («toi, qui es-tu?») qui cerne l'identité de Poimandrès, avec le nom qui couronne le tout ( $\mathrm{CH}$ I 2): «Moi, je suis Poimandrès, l'Intellect de la Souveraineté; je sais ce que tu veux et je suis avec toi partout.»

L'homophonie avec poimèn exploitée par plusieurs auteurs antiques et modernes n'est pas incompatible avec une étymologie antérieure, égyptienne, qui aurait été la principale raison du choix ou de l'invention du nom par l' auteur. Que le lecteur puisse difficilement comprendre la signification exacte du nom ne justifie pas de mettre en avant uniquement une étymologie grecque. En effet, qu'un nom

47. C'est l'opinion de Walter Scott, Hermetica, op. cit., vol. 2, p. 15.

48. Voir en particulier $C H$ I 22 qui décrit l'attitude de Poimandrès auprès des hommes saints, purs et bons. 
soit incompréhensible à la majorité pouvait être perçu comme un gage d'un savoir théologique authentique ${ }^{49}$. De plus, l'auteur de l'écrit n'avait pas l'intention de s'adresser à tous les hommes, mais aux seuls hommes les plus dignes, selon l'injonction de Poimandrès au narrateur à la fin de la révélation ( $\mathrm{CH}$ I 26) : «Ayant tout hérité, ne vas-tu pas devenir un guide pour les (hommes) dignes, afin que le genre de l'humanité, par ton entremise, soit sauvé par Dieu?» Enfin, il nous semble que ce texte ne s'adresse pas à des novices mais à des disciples bien avancés; en effet, nous avons essayé de montrer ailleurs que ce texte pourrait être lié à un rituel d'investiture d'un disciple en maître hermétique, successeur d'Hermès ${ }^{50}$.

\section{LE RÔLE DU NOM DANS L'ÉCONOMIE DE LA RÉVÉLATION}

Au-delà de l'étymologie possible, le nom a un rôle dans l'économie à la fois de la révélation et du possible rituel d'investiture. Poimandrès intervient à plusieurs reprises dans $C H$ I et le plus souvent à des moments stratégiques, en particulier au début et à la fin de la révélation. Nous allons surtout détailler ces deux moments, qui correspondent respectivement à la première mention et aux deux dernières mentions du nom divin dans le traité.

\section{Poimandrès en $\mathrm{CH}$ I 1-4}

\section{L'auteur commence son écrit par ces mots ( $C H$ I 1-4):}

Un jour, alors qu'une réflexion m'était advenue au sujet des êtres et que j'avais élevé très haut la pensée, que mes sensations corporelles avaient été retenues, comme ceux alourdis par le sommeil à cause d'une satiété de nourriture ou à cause d'une lassitude du corps, il me sembla qu'un être très grand, d'une mesure illimitée se présenta, appelant mon nom et me disant:

«Que veux-tu écouter, contempler et, ayant réfléchi, apprendre et connaître?»

Je dis: «Toi, qui es-tu?»

49. Les papyrus magiques et les textes gnostiques transmettent de nombreux noms dits «magiques» qui ne sont pas directement compréhensibles pour un noninitié.

50. Cf. Anna Van den Kerchove, La Voie d'Hermès. Pratiques rituelles et traités hermétiques, Leyde, Brill, 2012 («Nag Hammadi and Manichaean Studies» 77), p. 44-45. 
«Je suis», dit-il, «Poimandrès, l'Intellect de la Souveraineté; je sais ce que tu veux et je suis avec toi partout.»

Je dis: «Je désire apprendre sur les êtres, comprendre leur nature et connaître Dieu. Comme je veux écouter!» dis-je.

Il me dit à nouveau: "Garde dans ton intellect ce que tu désires apprendre et moi, je t'enseignerai.»

Ayant dit ceci, il changea de forme et aussitôt tout s'ouvrit devant moi en un moment, et je vis une vision sans limite, tout étant devenu Lumière.

Le début du traité met en place le statut divin de la révélation cosmologique et anthropologique qui suit. Il le fait, non pas en utilisant une proposition telle que «les paroles transmises par les dieux», ni en adoptant la forme poétique - considérée comme le signe d'un caractère divin ${ }^{51}$-, mais en ayant recours à une construction littéraire qui établit une analogie entre la réception de la révélation et l'obtention d'un oracle ${ }^{52}$. Il est en effet possible de retrouver les trois étapes oraculaires: les préparatifs de celui qui sollicite l'oracle, la question posée et la réponse divine qui débute par l'auto-présentation de la divinité - ce qui est fréquent dans les réponses oraculaires ${ }^{53}$. La construction littéraire établit également un contraste entre les deux protagonistes : le narrateur, ignorant - il cherche mais ne trouve pas - et marqué par la corporéité qu'il doit mettre en sommeil temporairement; l'être épiphanique, que ses qualités physiques et cognitives ${ }^{54}$ désignent comme un être divin. Au sommet de la construction, se trouve le nom, dont la rareté devait impressionner le lecteur et qui devait lui évoquer les idées du berger, de la vigilance et de la sollicitude. Ces idées reviennent plusieurs fois dans le texte, avec la mention régulière du nom Poimandrès et avec des affirmations identitaires «je suis avec toi partout» $(\mathrm{CH} \mathrm{I}$ 4), et «gardien des portes» (pulôros, CH I 22). La mention du nom divin renforce le contraste entre l'être divin et le narrateur. Le premier se nomme et son nom est transmis par l'écrit; le second

51. Pseudo-Longin, Sur le Sublime 8.4; Plutarque, Les Oracles de la pythie, 396 C-D, 402 B et 406 F-407 A.

52. Cf. Anna Van den Kerchove, «Le mode de révélation dans les Oracles chaldaïques et dans les traités hermétiques », dans Helmut Seng et Michel Tardieu, Die Chaldaeischen Orakel: Kontext - Interpretation - Rezeption, Heidelberg, Universitätsverlag Winter GmbH, 2011 («Bibliotheca Chaldaica»), p. 145-162.

53. David E. Aune, Prophecy in Early Christianity and the Ancient Mediterranean World, Grand Rapids (Michigan), Eerdmans, 1983, p. 64.

54. Voir plus haut p. 29. 
est nommé par la divinité mais son nom est tu dans l'écrit. Ce qui importe dans un cas est le nom lui-même, dans l'autre, le fait d'être appelé, prélude au «recrutement religieux », à l'investiture comme guide salvateur qui intervient à la fin de la révélation ( $C H$ I 26). Il s'agit véritablement d'une vocation religieuse qui est authentifiée non pas par une institution, mais par la mention du nom divin.

\section{Poimandrès en $\mathrm{CH} \mathrm{I} 30$}

Les deux dernières mentions du nom divin se trouvent à la fin de la révélation apocalyptique au moment où Poimandrès se dérobe aux yeux du narrateur pour se mêler aux Puissances divines et juste avant que le narrateur n'adresse un hymne au Dieu Père (CH I 30) :

Et quant à moi, je gravai en moi-même le bienfait de Poimandrès, et, étant rempli de ce que je souhaitais, je me réjouissais beaucoup. En effet, le sommeil du corps était devenu sobriété de l'âme, la fermeture des yeux une vue véritable, mon silence plein de bien et l'expression de la parole, des générations de bonnes choses. Ceci m'arriva, l'ayant reçu de mon intellect, c'est-à-dire Poimandrès, la Parole de la Souveraineté.

La dernière occurrence fait écho à la première mention au début du traité, avec une construction syntaxique identique: nom divin et expression apposée au nom. Seule l'expression change: il s'agit désormais de la «parole de la Souveraineté». Dans les deux cas, le nom divin est associé à authentia, qui désigne «le plein pouvoir» ou «l'autorité » ${ }^{55}$. Dans des écrits chrétiens considérés comme hérétiques, le terme désigne Dieu, le monde divin ou le démiurge ${ }^{56}$. Dans d'autres écrits chrétiens, il qualifie des écrits, comme chez Clément d'Alexandrie qui affirme que les Écritures sont «fortes d'une autorité absolue et toute-puissante ${ }^{57}$. Dans le cas hermétique, le terme désigne le Dieu très haut, qui correspondrait au dieu égyptien Rê. De même que l'autorité des «livres de Thot» provient de Rê par l'intermédiaire de Thot, son secrétaire et remplaçant

55. Pierre Chantraine, Dictionnaire étymologique de la langue grecque, vol. 1, Paris, Klincksieck, 1968, p. 138. Voir aussi Francisco R. Adrados (dir.), Diccionario Griego-Espanol, vol. 3, Consejo superior de investigaciones científicas, Instituto de Filología, Madrid, 1991, p. 602.

56. Jörg Büchli, Poimandres. Ein paganisiertes Evangelium, Tübingen, MohrSiebeck, 1987, p. 21-24. Élenchos VII 28.2 (pour Satornil) et VII 33.3 (pour Cérinthe); Hypostase des Archontes, NH II 94.

57. Clément d'Alexandrie, Stromates IV, I 2, 2. 
en son absence, de même l'autorité de la révélation hermétique provient de la Souveraineté par l'intermédiaire de Poimandrès, un aspect ${ }^{58}$ du Dieu très haut, dans le (seul ?) but d'instruire et de faire une révélation à celui dont la vocation est d'être le premier maillon de la chaîne hermétique. La position stratégique du nom divin avec une expression comportant le mot peu courant authentia au début et à la fin de la révélation revient à enchâsser la révélation apocalyptique dans un filet d'autorité divine.

Le nom Poimandrès, comme le nom Thot pour les «livres de Thot», est le garant de la légitimité de la révélation, et de ses conséquences pour le narrateur: son investiture comme guide salvateur ( $\mathrm{CH}$ I 26), qui répond à l'appel divin au début du traité et qui apparaît ainsi comme une vocation religieuse, et sa transformation intérieure, dont la description ( $\mathrm{CH}$ I 30) est encadrée par les deux dernières mentions du nom divin. Or, $\mathrm{CH}$ I pourrait être considéré comme un rituel d'investiture ${ }^{59}$ : l'anonymat aurait pour but de permettre d'identifier, dans le cadre d'une utilisation rituelle du texte, des disciples historiques au narrateur et de les instituer maîtres à l'instar de ce dernier; ces disciples seraient ainsi eux aussi les destinataires et les héritiers de la révélation divine, devenant maîtres non par cooptation, ni par apprentissage ni par d'autres modalités que l'on pourrait qualifier d'humaines, mais par vocation, par élection divine.

\section{Poimandrès dans $\mathrm{CH}$ XIII 15}

L'idée du nom divin comme garant de légitimité est à nouveau présente sous la plume de l'auteur du treizième traité hermétique. Seul autre auteur hermétiste à mentionner le nom divin, il le fait deux fois dans un même passage, $\mathrm{CH}$ XIII 15 :

$\{\mathrm{T}\}$ Père, je désirerai cette louange en forme d'hymne dont tu dis qu'on l'entendra des Puissances alors que je parviendrai à l'Ogdoade.

$\{\mathrm{H}\}$ Selon que Poimandrès révéla l'Ogdoade, enfant, tu (fais) bien de te hâter de briser la tente; car tu es pur. Poimandrès, l'Intellect de la Souveraineté, ne m'a pas transmis plus que ce qui est écrit, sachant que par moi-même, je serai capable de tout concevoir, d'entendre ce que je veux et de voir toutes les choses.

58. Sur l'utilisation du terme «aspect» pour la théologie hermétique: Alessandra Borgia, «Unita, unicita, totalita di dio nell'ermetismo antico», Studi e materiali di Storia delle religioni, n. s. 13.2, vol. 55, 1989, p. 197-211, part. p. 200-202.

59. Voir supra, note 50. 
Ce passage est stratégique dans la formation du disciple Tat par Hermès Trismégiste. Tat vient d'être entièrement régénéré, les Puissances divines l'ont investi, chassant les vices qui étaient jusque-là présents en lui. Il demande alors à bénéficier d'un hymne secret, l'hymne de l'Ogdoade, qui atteste que l'hermétiste a désormais atteint la sphère divine qu'est l'Ogdoade. Hermès ne répond pas immédiatement de façon positive à cette demande. Avant de donner des indications corporelles sur la manière de dire l'hymne et de l'entonner, il rappelle la chaîne de transmission dans laquelle il s'insère et il met l'accent sur celui qui transmet, Poimandrès. Ce nom est mentionné deux fois, et la seconde fois l'expression «l'Intellect de la Souveraineté» lui est apposée. Il est sujet de paradidômi, terme technique pour la transmission chez les Grecs, les chrétiens et dans les cultes à mystères ${ }^{60}$ et qui désigne peu à peu la tradition et en particulier l'enseignement secret ${ }^{61}$. Ce rappel - l'impression ressort que le disciple doit déjà connaître l'identité du maître d'Hermès - permet à Hermès de réaffirmer sa position d'héritier de Poimandrès et sa légitimité à chanter l'hymne de l'Ogdoade. Comme dans le premier traité, le nom divin en $\mathrm{CH}$ XIII apparaît comme un label d'authenticité.

\section{Conclusion}

Le nom divin Poimandrès paraît bien être au cœur d'une stratégie de l'auteur de $\mathrm{CH}$ I visant à établir l'autorité divine de la révélation qu'il transmet par écrit. Le sens du nom a sûrement joué dans le choix (ou l'invention) de ce nom. Si l'on accepte une étymologie égyptienne et copte, la mention du nom divin permettait à l'auteur de s'insérer ou d'affirmer son appartenance à la tradition de la sagesse égyptienne. Les lecteurs du texte ont-ils perçu cette étymologie? Si aucune donnée n'en atteste, cela ne semble pas être une raison

60. Walter Burkert, Les Cultes à mystères dans l'Antiquité, traduction Bernard Deforge et Louis Bardollet, Paris, Les Belles Lettres, 2003 («Vérité des mythes»), p. 65.

61. Eduard Norden, Agnostos Theos: Untersuchungen zur Formengeschichte religiöser Rede, Berlin, Teubner, 1913, p. 290. Le P. Berol. 21 196, ou Catéchisme mithraïque, serait «a rare example of an actual ancient text in the paradosis»; voir William M. Brashear, «A Mithraic Catechism from Egypt», Tyche. Supplementband, Wien, Verlag Adolf Holzhausens, 1992, p. 47. 
suffisante pour exclure une étymologie égyptienne, l'auteur visant un lectorat cultivé appartenant probablement en partie à un milieu de sagesse égyptienne. Toutefois, une étymologie grecque, secondaire, est possible, comme plusieurs témoignages antiques l'attestent. L'auteur de $C H$ I pourrait même avoir choisi (ou inventé) ce nom en partie en raison de l'homophonie avec poimèn.

Le nom divin, signature qui instaure une « autorité énonciative ${ }^{62}$, permet de légitimer et de fournir un label d'authenticité divine au contenu de ce qui est présenté comme une révélation divine et à ses conséquences. Il faut replacer cette stratégie dans le contexte religieux des $\mathrm{II}^{\mathrm{e}}-\mathrm{III}^{\mathrm{e}}$ siècles, où il existe ce que l'on pourrait appeler une véritable concurrence d' autorités, plusieurs traditions religieuses prétendant détenir leur savoir de très haut et dénigrant parfois celui des adversaires ${ }^{63}$. La révélation est ainsi présentée comme une voie de salut véridique, et le narrateur et ses héritiers historiques comme des élus dont la vocation religieuse est d'être les véritables guides salvateurs. Au moins une partie de la tradition hermétique, représentée par l'auteur de $\mathrm{CH}$ XIII, revendique explicitement ce fondement et reprend la même stratégie.

Les auteurs de $C H$ I et de $C H$ XIII n'agiraient pas autrement que plusieurs auteurs contemporains et ultérieurs, notamment les auteurs de certains traités transmis par les codices coptes découverts près de Nag Hammadi. Il serait intéressant de poursuivre l'étude en comparant avec ces autres documents.

Anna.van_den_kerchove@ephe.sorbonne.fr

Institut européen en sciences des religions

4-14 rue Ferrus, 75014 Paris

62. Gérard Leclercq, Histoire de l'autorité, op. cit., p. 6.

63. Cette concurrence, ou même ce conflit, est bien mise en évidence avec la polémique anti-chrétienne d'auteurs comme Celse et Porphyre et la polémique chrétienne avec Clément d'Alexandrie et Origène: Giulia Sfameni Gasparro, «Ispirazione delle Scritture e Divinazione Pagana Aspetti della Polemica fra Origene e Celso », dans Gilles Dorival et Alain Le Boulluec, Origeniana Sexta. Origène et la Bible / Origen and the Bible. Actes du Colloquium Origenianum Sextum. Chantilly 1993, Louvain, University Press, 1995 («Bibliotheca ephemeridum theologicarum Lovaniensium» CXVIII), p. 287-302. Un conflit analogue se déroule entre les chrétiens orthodoxes et les gnostiques: Elaine Pagels, "Visions, Appearances, and Apostolic Authority: Gnostic and Orthodox Traditions», dans Barbara Aland (éd.), Gnosis. Festschrift für Hans Jonas, Göttingen, Vandenhoeck \& Ruprecht, 1978, p. 415-430; et Christopher Tuckett, The Gospel of Mary, Oxford, Oxford University Press, 2007 («Oxford Early Christian Gospel Texts»), p. 200-201. 